\title{
Confinement and renormalization group equations in string-inspired nonlocal gauge theories
}

\author{
Marco Frasca $\odot,{ }^{1, *}$ Anish Ghoshal $\odot,{ }^{2,3, \dagger}$ and Nobuchika Okada $\odot^{4, \hbar}$ \\ ${ }^{1}$ Rome, Italy \\ ${ }^{2}$ INFN_Sezione Roma Tor Vergata, Via della Ricerca Scientifica 1, 00133 Rome, Italy \\ ${ }^{3}$ Institute of Theoretical Physics, Faculty of Physics, University of Warsaw, \\ ulica Pasteura 5, 02-093 Warsaw, Poland \\ ${ }^{4}$ Department of Physics and Astronomy, University of Alabama, \\ Tuscaloosa, Alabama 35487, USA
}

(Received 10 July 2021; accepted 29 September 2021; published 16 November 2021)

\begin{abstract}
As an extension of weak perturbation theory obtained in a recent analysis of infinite-derivative nonlocal non-Abelian gauge theories motivated from $p$-adic string field theory, and postulated as a direction of UV completion in four-dimensional quantum field theory, here we investigate the confinement conditions and $\beta$ function in the strong coupling regime. We extend the confinement criterion, previously obtained by Kugo and Ojima for the local theory based on the Becchi-Rouet-Stora-Tyutin invariance, to the nonlocal theory by using a set of exact solutions of the corresponding local theory. We show that the infinite derivatives which are active in the UV provide finite contributions also in the infrared limit and provide a proof of confinement, granted by the absence of the Landau pole. We also show that in the limit of the energy scale of nonlocality $M \rightarrow \infty$ we reproduce the local theory results and see how asymptotic freedom is properly recovered.
\end{abstract}

DOI: 10.1103/PhysRevD.104.096010

\section{INTRODUCTION}

In the context of $p$-adic string field theory, recently, higher-derivative approaches to a UV completion of quantum field theory (QFT) have become popular. Initially, they were proposed as possible UV-regularized theories [1-6]. In this context, the infinite higher-derivative approach was motivated starting from string field theory [7-21], where attempts were made to address the divergence problem by generalizing the kinetic energy operators of the Standard Model (SM) to an infinite series of higher order derivatives suppressed by the scale of nonlocality $(M)$ at which the higher order derivatives come into the picture $[22,23]$.

Such a theoretical construction naturally solves the SM vacuum instability problem as the $\beta$ functions at the scale of nonlocality vanish beyond $M$, without introducing any new degrees of freedom in the particle spectrum [24]. They have been explicitly shown to be ghost-free [25], predicting conformal invariance in the UV, trans-Planckian scale

\footnotetext{
*marcofrasca@mclink.it

†anish.ghoshal@roma2.infn.it

okadan@ua.edu
}

Published by the American Physical Society under the terms of the Creative Commons Attribution 4.0 International license. Further distribution of this work must maintain attribution to the author(s) and the published article's title, journal citation, and DOI. Funded by SCOAP. transmutation and dark matter phenomenology [26,27]. This approach is a novel direction toward UV completion of four-dimensional QFT, valid and perturbative up to infinite energy scales without Landau-pole problems $[24,26]$. Strong coupling regimes of the theory were studied in Refs. [28,29], where it was shown that the mass gap obtained gets diluted in the UV due to nonlocal effects restoring conformal invariance in the UV.

Gravity theories with such an infinite-derivative approach have been investigated in Ref. [30]. In the context of most general quadratic curvature gravitational action (parity invariant and torsion-free), it was shown that it is possible to make the gravitational sector free from the Weyl ghost and from any classical singularities, like black-hole singularities [30-41] and cosmological singularities [42-47].

Generally speaking, we employ the renormalization group equations (RGEs) to understand the relevance of UV fixed points for quantum field theories $[48,49]$. For example, in quantum chromodynamics, the property known as the asymptotic freedom shows the reliability of the theory by the use of the standard perturbation theory [50,51]. Asymptotic freedom is missing in both the $\mathrm{U}(1)$ and the scalar sector of the Standard Model, which is the so-called triviality problem, limiting the understanding of the behavior of the theory at very large energies [52]. When the fixed point corresponds to an interacting theory, we 
speak of asymptotic safety [53]. It would be fine for theories that are neither asymptotically free nor renormalizable to have such a UV fixed point [54]. This idea was recently developed for quantum gravity [54-60]. Applications to the Standard Model also showed such a possibility [61-63]. The authors studied the RGEs of nonlocal theories in Refs. [24,64] but only in the weak coupling regime. In this paper, we extend the same approach to the strong coupling regime by employing a novel technique which we will describe below.

Besides the RGE approach in QFT, a deeper understanding of the confinement of quarks in the Standard Model (QCD sector) has eluded us for many years, with some possible mechanism existing in the literature (see [65] and references therein). Kugo and Ojima proposed a confinement condition from the Becchi-Rouet-StoraTyutin (BRST) invariance based on charge annihilation $[66,67]$. On similar grounds, Nishijima and collaborators [68-72] derived constraint on the amplitudes of unphysical states leading to color confinement merely as a consequence of the BRST invariance and asymptotic freedom of the QCD theory. ${ }^{1}$ Confinement, in its simplest form, can be understood as the combined effect of a potential obtained from the Wilson loop of a Yang-Mills theory without fermions and the running coupling yielding a linearly incrementing potential, in agreement with lattice data [90].

In this paper, we will apply the condition derived in [68-72] (by reducing it to the case of the Kugo-Ojima criterion [67]), nonlocal non-Abelian gauge theories without fermions, as we begin with the known exact solutions $[28,29,88]$. We will prove that nonlocal non-Abelian gauge theory with no fermions is confining in four dimensions, besides having a mass gap coming from the derived correlation functions. This is purely because confinement arises due to the BRST invariance and the asymptotic freedom of the theory, as well as the existence of a mass gap. We show that the infinite-derivative operators defined in the UV yield finite contributions also in the IR limit and provide a proof of confinement granted by the absence of the Landau pole.

\footnotetext{
${ }^{1}$ In supersymmetric models, confinement is proven in certain conditions as a condensation of monopoles, similar to type II superconductors $[73,74]$. For a comparison of different confinement theories and their overlapping regions, see [75]. Besides, studies by Gribov [76] and Zwanziger [77] suggested confinement in QCD with the gluon propagator running to zero as momenta go to zero and an enhanced ghost propagator running to infinity more rapidly than the free case in the same limit of momenta. Such a scenario does not agree with the mass gap appearance from studies of the gluon and ghost propagators on the lattice [78-80] and the spectrum [81,82] in non-Abelian gauge theories without fermions. These results found a theoretical basis in Refs. [83-88] in terms of a closed form formula for the gluon propagator (see Ref. [89] for a review and other results for the running coupling in the infrared limit besides the gluon and ghost propagators).
}

The paper is organized as follows: In Sec. II, we introduce the nonlocal Yang-Mills theory for $\mathrm{SU}(\mathrm{N})$, starting with the well-known setting of the local theory. In Sec. III, we extend the formalism of BRST invariance of the local theory to the nonlocal case and the Kugo-Ojima confinement criterion to the latter. In Sec. IV, we evaluate explicitly the Kugo-Ojima criterion for the nonlocal case and, in Sec. V, we derive the beta function for the nonlocal theory that holds in the infrared limit. Finally, in Sec. VI, some discussion and conclusions are presented.

\section{INFINITE-DERIVATIVE NON-ABELIAN GAUGE THEORIES: REVIEW}

The Lagrangian for the SU(N) pure Yang-Mills theory, in the local case, takes the form

$$
\mathcal{L}_{g}=-\frac{1}{4} F^{a \mu \nu} F_{a \mu \nu}
$$

Repeated indices imply summation both for space-time and group indices. The field strength tensor is given by

$$
F_{\mu \nu}^{a}=\partial_{[\mu} A_{\nu]}^{a}-g f^{a b c} A_{\mu}^{b} A_{\nu}^{c},
$$

with the group structure constants $f^{a b c}$ and the dimensionless gauge coupling $g$. We extend the theory to the nonlocal case by following the approach given in Refs. [24,64]. A common definition involving infinite series of higher derivatives yielded in the literature [22-24,26,64]

$$
L_{f}=-\frac{1}{4} F_{\mu \nu}^{a} e^{-f\left(D^{2}\right)} F^{a \mu \nu} .
$$

Let us take

$$
f\left(D^{2}\right)=\frac{D^{2}}{M^{2}},
$$

where $D_{\mu}^{a b}=\partial_{\mu} \delta^{a b}-i g A_{\mu}^{c}\left(T^{c}\right)^{a b}$ is the covariant derivative in the adjoint representation. We have introduced a mass scale $M$ for the scale of nonlocality. The scale is assumed to be large since nonlocal effects have not been experimentally observed so far. This implies that the variation in momentum scale of the $D^{2}$ is lower than $M^{2}$. For

$$
\begin{aligned}
D^{2} & =\left(\partial_{\mu}-i g T^{a} A_{\mu}^{a}\right)^{2} \\
& =\partial^{2}-i g \partial^{\mu}\left(T^{a} A_{\mu}^{a}\right)-i g T^{a} A_{\mu}^{a} \partial^{\mu}-g^{2} T^{a} T^{b} A_{\mu}^{a} A^{b \mu},
\end{aligned}
$$

one can apply the Baker-Campbell-Hausdorff formula,

$$
\begin{aligned}
e^{-\frac{D^{2}}{M^{2}}}= & e^{-\frac{\square}{M^{2}}} e^{-\frac{1}{M^{2}}\left(-i g \partial^{\mu}\left(T^{a} A_{\mu}^{a}\right)-i g T^{a} A_{\mu}^{a} \partial^{\mu}-g^{2} T^{a} T^{b} A_{\mu}^{a} B^{b \mu}\right)} \\
& \times e^{-\frac{1}{2 M^{4}}\left[\square,-i g \partial^{\mu}\left(T^{a} A_{\mu}^{a}\right)-i g T^{a} A_{\mu}^{a} \partial^{\mu}-g^{2} T^{a} T^{b} A_{\mu}^{a} b^{b \mu}\right]} \times \ldots,
\end{aligned}
$$


and then the commutators are all higher orders with respect to the first two exponentials in the formula. Assuming that the field amplitudes are always negligibly small with respect to the nonlocality scale $M$, we can write

$$
L_{f} \sim-\frac{1}{4} F_{\mu \nu}^{a} e^{-f(\square)} F^{a \mu \nu} .
$$

This affects gauge invariance but the effect turns out to be irrelevant, as the contribution arising from a change in the gauge potentials will produce higher order terms with respect to the nonlocality scale. This means that we can assume Eq. (7) with a negligible effect on our results. Such an argument is perfectly consistent with the computation reported in [3], where the nonlocal factors in the propagators are evaluated using $\square$ rather than $D^{2}$. The conventional Lagrangian is reproduced in the limit of $M \rightarrow \infty$. We take the metric convention with $\operatorname{diag}(+1,-1,-1,-1)$ to implement our procedure for UV completion upon the Wick rotation. $^{2}$

\section{A. Gauge field redefinition}

Let us define

$$
\hat{A}_{\mu}^{a}=e^{-\frac{1}{2} f(\square)} A_{\mu}^{a}
$$

The idea with this change of variables is to eliminate the exponential nonlocal factor from the kinetic term just as happens for scalar field theory. Indeed, our work will parallel the one already presented in [28]. With the redefinition, we will arrive at the Lagrangian

$\mathcal{L}_{f}=\frac{1}{2} \hat{A}_{\mu}^{a}\left(\square \eta^{\mu \nu}-\partial^{\mu} \partial^{\nu}\right) \hat{A}_{\nu}^{a}$

$-\frac{g}{4} f^{a b c} e^{-f(\square)}\left[e^{\frac{1}{2} f(\square)}\left(\partial_{\mu} \hat{A}_{\nu}^{a}-\partial_{\nu} \hat{A}_{\mu}^{a}\right)\left(e^{\frac{1}{2} f(\square)} \hat{A}^{b \mu} e^{\frac{1}{2} f(\square)} \hat{A}^{c \nu}\right)\right]$

$-\frac{g}{4} f^{a b c} e^{-f(\square)}\left[e^{\frac{1}{2} f(\square)} \hat{A}^{b \mu} e^{\frac{1}{2} f(\square)} \hat{A}^{c \nu} e^{\frac{1}{2} f(\square)}\left(\partial_{\mu} \hat{A}_{\nu}^{a}-\partial_{\nu} \hat{A}_{\mu}^{a}\right)\right]$

$-\frac{g^{2}}{4} f^{a b c} f^{c d e} e^{-f(\square)}\left[e^{\frac{1}{2} f(\square)} \hat{A}^{b \mu} e^{\frac{1}{2} f(\square)} \hat{A}^{c \nu} e^{\frac{1}{2} f(\square)} \hat{A}_{\mu}^{d} e^{\frac{1}{2} f(\square)} \hat{A}_{\nu}^{e}\right]$

$+j_{\mu}^{a} e^{\frac{1}{2} f(\square)} \hat{A}^{a \mu}$,

where we added an arbitrary source term $j_{\mu}^{a}$ that will be useful in the following. This is similar to the formulation of the nonlocal scalar field theory. The main difference is the multiplication of the interaction part by the nonlocal factor $e^{-f(\square)}$.

\footnotetext{
${ }^{2}$ We will work in the Euclidean space with a certain relation between Minkowski and Euclidean metrics assuming the conclusions given in [91-94], which are done via analytical continuation. Practically, at least in our computations, it is fine to use the standard Wick rotation.
}

The non-Abelian ghost and gauge-fixing Lagrangians are given by

$$
\mathcal{L}_{\text {ghost }}=-\bar{c}^{a} e^{-f(\square)}\left(\partial^{\mu} D_{\mu}^{a b}\right) c^{b}
$$

and

$$
\mathcal{L}_{g-f}=\frac{1}{2 \xi} \hat{A}_{\mu}^{a} e^{-f(\square)} \partial^{\mu} \partial^{\nu} \hat{A}_{\nu}^{a}
$$

where $\xi$ is the gauge-fixing parameter. In order to have consistency with the standard gauge-fixing procedure, we choose the entire function $e^{-f(\square)}$. The standard QFT result can be obtained in the local limit of $M \rightarrow \infty$.

Similar to what we have done for the gauge field, the ghost field can be redefined as

$$
c^{a}=e^{-\frac{1}{2} f(\square)} \hat{c}^{a} .
$$

This will yield

$$
\begin{aligned}
\mathcal{L}_{\text {ghost }}= & -\overline{\hat{c}}^{a} \partial^{\mu}\left(\partial_{\mu} \delta^{a b}-i g e^{\frac{1}{2} f(\square)} \hat{A}_{\mu}^{c}\left(T^{c}\right)^{a b}\right) \hat{c}^{b} \\
& +\bar{\eta}^{a} e^{\frac{1}{2} f(\square)} \hat{c}^{a}+e^{\frac{1}{2} f(\square)} \overline{\hat{c}}^{a} \eta .
\end{aligned}
$$

Also in this case, we added arbitrary source terms $\eta^{a}$ and $\bar{\eta}^{a}$.

Finally, our Lagrangian is given by

$$
\mathcal{L}=\mathcal{L}_{f}+\mathcal{L}_{g-f}+\mathcal{L}_{\text {ghost }}
$$

\section{CONFINEMENT AND BRST INVARIANCE}

We briefly describe the condition of confinement derived in [68-72], and then we reduce it to the case of the KugoOjima criterion [67], for nonlocal non-Abelian gauge theories without fermions, as we begin with known exact solutions $[28,29,88]$.

\section{A. Local theory}

Here we present the notations and the formalism as used in [95] for confinement conditions in local non-Abelian theories.

For the Yang-Mills field we have the following Lagrangian:

$$
\mathcal{L}=\mathcal{L}_{\text {inv }}+\mathcal{L}_{g f}+\mathcal{L}_{\mathrm{FP}}
$$

Here, we have described $\mathcal{L}$ with $\mathcal{L}_{\text {inv }}$ for the classical gauge-invariant part, $\mathcal{L}_{g f}$ for the gauge-fixing terms and $\mathcal{L}_{\mathrm{FP}}$ for the Faddeev-Popov (FP) ghost term proper to nonAbelian gauge theories: 


$$
\begin{aligned}
& \mathcal{L}_{\text {inv }}=-\frac{1}{4} F_{\mu \nu} \cdot F^{\mu \nu}, \\
& \mathcal{L}_{g f}=\partial_{\mu} B \cdot A^{\mu}+\frac{1}{2} \xi B \cdot B, \\
& \mathcal{L}_{\mathrm{FP}}=i \partial_{\mu} \bar{c} \cdot D^{\mu} c,
\end{aligned}
$$

where $\xi$ denotes the gauge parameter and $D_{\mu}$ is the covariant derivative given by

$$
\begin{aligned}
D_{\mu} \psi & =\left(\partial_{\mu}-i g T \cdot A_{\mu}\right) \psi, \\
D_{\mu} c^{a} & =\partial_{\mu} c^{a}+g f^{a b c} A_{\mu}^{b} c^{c} .
\end{aligned}
$$

As done in [66], we introduce the BRST transformations of a generic field $\chi$ using the BRST charges $Q_{B}$ and $\bar{Q}_{B}$ given by

$$
\begin{gathered}
\delta \chi=i\left[Q_{B}, \chi\right]_{\mp}, \quad \bar{\delta} \chi=i\left[\bar{Q}_{B}, \chi\right]_{\mp}, \\
Q_{B}^{2}=\bar{Q}_{B}^{2}=Q_{B} \bar{Q}_{B}+\bar{Q}_{B} Q_{B}=0 .
\end{gathered}
$$

We will take the minus (plus) sign in (20) when $\chi$ is even (odd) in the ghost fields $c$ and $\bar{c}$. These are anticommuting scalar fields.

The BRST transformations of the gauge field $A_{\mu}$ are generally defined by replacing the infinitesimal gauge function by the FP ghost field $c$ or $\bar{c}$, in their respective infinitesimal gauge transformations

$$
\begin{aligned}
& \delta A_{\mu}=D_{\mu} c, \\
& \bar{\delta} A_{\mu}=D_{\mu} \bar{c} .
\end{aligned}
$$

By imposing for the auxiliary fields $B, c$ and $\bar{c}$

$$
\delta \mathcal{L}=\bar{\delta} \mathcal{L}=0,
$$

one gets

$$
\begin{aligned}
& \delta B=0, \quad \delta \bar{c}=i B, \quad \delta c^{a}=-\frac{1}{2} g f^{a b c}\left(c^{b} c^{c}\right), \\
& \bar{\delta} \bar{B}=0, \quad \bar{\delta} c=i \bar{B}, \quad \bar{\delta} \bar{c}^{a}=-\frac{1}{2} g f^{a b c}\left(\bar{c}^{a} \bar{c}^{c}\right),
\end{aligned}
$$

with $\bar{B}$ defined by the following equation:

$$
B^{a}+\bar{B}^{a}-i g f^{a b c}\left(c^{b} \bar{c}^{c}\right)=0 .
$$

From Noether's theorem (up to a total divergence) one gets the conserved current

$$
\begin{aligned}
j_{\mu} & =\sum_{\{\Phi\}} \frac{\partial L}{\partial\left(\partial_{\mu} \Phi\right)} \delta \Phi \\
& =B^{a}\left(D_{\mu} c\right)^{a}-\partial_{\mu} B^{a} c^{a}+i \frac{1}{2} \mathrm{~g} f^{a b c} \partial_{\mu} \bar{c}^{a} c^{b} c^{c},
\end{aligned}
$$

with $\{\Phi\}$ being the set of all fields present in the Lagrangian. Therefore, the corresponding charge $Q_{B}$ is given by

$Q_{B}=\int d^{3} x\left(B^{a}\left(D_{0} c\right)^{a}-\dot{B}^{a} c^{a}+i \frac{1}{2} \mathrm{~g} f^{a b c} \dot{\bar{c}}^{a} c^{b} c^{c}\right)$.

Finally, the full Lagrangian will give

$$
\delta\left(\mathcal{L}_{g f}+\mathcal{L}_{\mathrm{FP}}\right)=\delta\left(-i \partial_{\mu} \bar{c} \cdot A_{\mu}-\frac{i}{2} \xi \bar{c} \cdot B\right),
$$

confirming that

$$
\delta \mathcal{L}_{\text {inv }}=0 .
$$

From this Lagrangian we immediately obtain the equations of motion

$$
D^{\mu a b} F_{\mu \nu}^{b}+j_{\nu}^{a}=i \delta \bar{\delta} A_{\nu}^{a} .
$$

On the right-hand side, we see the contributions coming from the auxiliary fields. These are massless particles at the tree level. We also note that the $B$ field does not propagate. The consequences of this is that such fields will not give any contribution to the physical spectrum of the theory. Besides, being $\partial^{\nu}\left(i \delta \bar{\delta} A_{\nu}\right)=0$, such a current is conserved. In order to evaluate such a contribution, we have to compute

$$
\left\langle i \delta \bar{\delta} A_{\mu}^{a}(x), A_{\nu}^{b}(y)\right\rangle .
$$

Referring to Kuog-Ojima formalism, we note that

$$
\delta \bar{\delta} A_{\mu}^{a}=-\left\{Q_{B},\left\{\bar{Q}_{B}, A_{\mu}^{a}\right\}\right\} .
$$

Then, because of $\left\langle 0\left|Q_{B}=Q_{B}\right| 0\right\rangle=\bar{Q}_{B}|0\rangle=\langle 0| \bar{Q}_{B}=0$, one has

$$
\begin{aligned}
\left\langle i \delta \bar{\delta} A_{\mu}^{a}(x), A_{\nu}^{b}(y)\right\rangle & =\left\langle i \bar{\delta} A_{\mu}^{a}(x), \delta A_{\nu}^{b}(y)\right\rangle \\
& =i\left\langle D_{\mu} \bar{c}^{a}(x), D_{\nu} c^{b}(y)\right\rangle .
\end{aligned}
$$

For this correlator, Kugo and Ojima showed [67] that

$$
\begin{aligned}
\int d^{d} x e^{i p x}\left\langle D_{\mu} \bar{c}^{a}(x), D_{\nu} c^{b}(y)\right\rangle= & \delta^{a b}\left(\delta_{\mu \nu}-\frac{p_{\mu} p_{\nu}}{p^{2}-i \epsilon}\right) u\left(p^{2}\right) \\
& -\delta^{a b} \frac{p_{\mu} p_{\nu}}{p^{2}-i \epsilon}
\end{aligned}
$$

and the no-pole condition yields here

$$
1+u\left(p^{2}=0\right)=0
$$

which is the Kugo-Ojima condition for confinement granting that no massless pole appears in the spectrum of the 
theory. Indeed, this condition removes the massless term from Eq. (33).

The function $u\left(p^{2}\right)$ was computed explicitly for nonAbelian gauge theories in [95], and the confinement was proven for such theories; moreover it also yields an exact beta function for the theory including the strong coupling regimes. Next, we will extend this computation to the nonlocal case.

\section{B. Nonlocal theory}

The complete BRST-invariant infinite-derivative gauge theory in the quantized action is of the form [64]

$$
\begin{aligned}
\mathcal{L}_{\text {inv }}= & -\frac{1}{4}\left(F_{\mu \nu}^{a} e^{-f\left(D^{2}\right)}\left(F^{a}\right)^{\mu \nu}\right) \\
& +\frac{\xi}{2}\left(B^{a}\right)^{2}+B^{a} \partial^{\mu} A_{\mu}^{a}+\bar{c}^{a}\left(-\partial^{\mu} e^{-\frac{1}{2} f(\square)} D_{\mu}^{a c}\right) c^{c},
\end{aligned}
$$

where $\xi$ is the gauge-fixing parameter, $B$ is the auxiliary field, and $c$ and $\bar{c}$ are the ghost and antighost fields, respectively. The BRST transformations for non-Abelian gauge theories express a residual symmetry of the effective action which remains after the original gauge invariance has been broken by the addition of the gauge-fixing and ghost action terms. Our BRST transformations are modified in the following way:

$$
\begin{aligned}
& \delta A_{\mu}=D_{\mu} c, \\
& \bar{\delta} A_{\mu}=D_{\mu} \bar{c},
\end{aligned}
$$

and

$$
\begin{aligned}
& \delta B=0, \quad \delta \bar{c}=i e^{\frac{1}{2} f(\square)} B, \quad \delta c^{a}=-\frac{1}{2} g f^{a b c}\left(c^{b} c^{c}\right), \\
& \bar{\delta} \bar{B}=0, \quad \bar{\delta} c=i e^{\frac{1}{2} f(\square)} \bar{B}, \quad \bar{\delta} \bar{c}^{a}=-\frac{1}{2} g f^{a b c}\left(\bar{c}^{b} \bar{c}^{c}\right) .
\end{aligned}
$$

We show the BRST invariance of $S_{\text {inv }}$ by noting that the BRST transformation of the gauge field is just a gauge transformation of $A_{\mu}$ generated by $c_{a}$ or $\bar{c}_{a}$. Therefore, any gauge-invariant functionals of $F_{\mu \nu}$, like the first term in Eq. (35), gives $\delta\left(-\frac{1}{4}\left(F_{\mu \nu}^{a} e^{-f\left(D^{2}\right)}\left(F^{\mu \nu}\right)^{a}\right)\right)=0$. The second term in Eq. (35) gives $\delta\left(\frac{\xi}{2}\left(B^{a}\right)^{2}\right)=0$ from Eq. (37). For the third term in Eq. (35), the transformation of $\mathrm{A}_{\mu}^{a}$ cancels the transformation of $\bar{c}$ in the last term due to Eqs. (36), leaving us with

$$
\delta\left(D_{\mu}^{a c} c^{c}\right)=D_{\mu}^{a c} \delta c^{c}+g f^{a b c} \delta A_{\mu}^{b} c^{c},
$$

which is is equal to 0, using the Jacobi identity (see Ref. [96]). The transformation of $c^{\sigma}$ is nilpotent,

$$
\delta\left(\partial_{\mu} c^{a} c^{b}\right)=0
$$

while the transformation of $A^{\mu}$ is also nilpotent,

$$
\delta\left(\left(D_{b}^{\mu}\right)^{a} c^{b}\right)=0
$$

Hence, the action in Eq. (35) is BRST invariant. Noting the fact that the only part of the ghost action which varies under the BRST transformations is that of the antighost $\left(\bar{c}_{a}\right)$, the central idea behind our proof of BRST invariance is that we have chosen the BRST variation of the antighost $\left(\bar{c}_{a}\right)$ [see Eqs. (37)] to cancel the variation of the gauge-fixing term.

It is not difficult to see that, in the limit of the nonlocal mass $M \rightarrow \infty$, the BRST transformations given in Eqs. (36) and (37) become identical to those of the local case. Formally, the confinement condition of Eq. (34) remains untouched, as the effects of the nonlocality, if present, are kept in the $u$ function.

\section{CONDITION OF CONFINEMENT IN NONLOCAL THEORY}

In this section, we derive the confinement for the nonlocal theory, following Ref. [95]. See Appendix C for a brief review of this technique.

From the action (35), we derive the equations of motion,

$$
e^{-f\left(D^{2}\right)} D^{\mu} F_{\mu \nu}^{a}+j_{\nu}^{a}=i \delta \bar{\delta} A_{\nu}^{a} .
$$

The rhs can be evaluated as already done for the local case, and we write down

$$
\begin{aligned}
\int d^{4} x e^{i p x}\left\langle D_{\mu} \bar{c}^{a}(x), D_{\nu} c^{b}(0)\right\rangle= & \delta_{a b}\left(\delta_{\mu \nu}-\frac{p_{\mu} p_{\nu}}{p^{2}}\right) u\left(p^{2}\right) \\
& -\delta_{a b} \frac{p_{\mu} p_{\nu}}{p^{2}} e^{f\left(-p^{2}\right)}
\end{aligned}
$$

Indeed, this is the most general form for the given correlation function but, for the massless contribution, we have also to take into account the contribution of the nonlocality. The interesting part here is that all the nonlocal contributions enter into the definition of the function $u$. These nonlocalities arise from the two-point functions of the nonlocal theory but also that fluctuations from UV can yield a significant contribution to confinement as they are summed up in the integral where they cannot be neglected. Then, the confinement condition is again

$$
1+u\left(p^{2}=0\right)=0 .
$$

To evaluate this equation, we will have for the two-point function [29] 


$$
G_{2}(p)=\frac{e^{\frac{1}{2} f\left(-p^{2}\right)}}{p^{2}+\Delta m^{2} e^{\frac{1}{2} f\left(-p^{2}\right)}} \frac{1}{1-\Pi(p)} .
$$

In the local limit, $M \rightarrow \infty$, Eq. (44) reduces to a Yukawa form that yields a fair approximation to the exact local propagator obtained in [88]. In the nonlocal case, one has the mass gap

$\Delta m^{2}=\mu^{2}\left(18 N g^{2}\right)^{\frac{1}{2}} \frac{4 \pi^{2}}{K^{2}(i)} \frac{e^{-\pi}}{\left(1+e^{-\pi}\right)^{2}} e^{f\left(-\frac{\pi^{2}}{4 K^{2}(i)} p^{2}\right)}+\delta m^{2}$.
This must be completed by the gap equation

$$
\delta m^{2}=2 N g^{2} G_{2}(0)=2 N g^{2} \int \frac{d^{4} p}{(2 \pi)^{4}} G_{2}(p) .
$$

The function $\Pi(p)$ can be neglected as also the shift $\delta m^{2}$ as a first approximation. Similarly, for the ghost one has

$$
K_{2}(p)=-\frac{1}{p^{2}} e^{\frac{1}{2} f\left(-p^{2}\right)}
$$

The confinement condition can be written as [95]

$$
\int d^{4} x e^{i p x}\left\langle D_{\mu} \bar{c}^{a}(x), D_{\nu} c^{b}(0)\right\rangle=-\delta^{a b} \frac{p_{\mu} p_{\nu}}{k^{2}}+\frac{\left(N^{2}-1\right)^{2}}{2 N} g^{2} \delta^{a b}\left(\delta_{\mu \nu}-\frac{p_{\mu} p_{\nu}}{p^{2}}\right) \int \frac{d^{4} p^{\prime}}{(2 \pi)^{4}} K_{2}\left(p-p^{\prime}\right) G_{2}\left(p^{\prime}\right) .
$$

This will yield for the confinement condition

$$
u(0)=-\frac{\left(N^{2}-1\right)^{2}}{2 N} g^{2} \int \frac{d^{4} p}{(2 \pi)^{4}} \frac{1}{p^{2}} \frac{e^{f\left(-p^{2}\right)}}{p^{2}+\Delta m^{2} e^{\frac{1}{2} f\left(-p^{2}\right)}} .
$$

From this integral, we see explicitly how the dependence on the nonlocality scale does appear. We will get contributions from the far UV that cannot be neglected as they sum up. Indeed, the nonlocal scale $M$ imposes a truncation in the spectrum and this turns out to be equivalent to a computation into infrared having singular UV behavior that cannot be neglected and a cutoff dependence pops out, like for Nambu-Jona-Lasinio models of low-energy QCD [97]. On the other hand, in local QCD, asymptotic freedom entails a dimensional transmutation with the appearance of a mass scale in the theory [50,51]. Indeed, in the local limit, $M \rightarrow \infty$, we are able to recover an approximate beta function by solving the integral

$$
u_{L L}(0)=-\frac{\left(N^{2}-1\right)^{2}}{2 N} g^{2} \int \frac{d^{4} p}{(2 \pi)^{4}} \frac{1}{p^{2}} \frac{1}{p^{2}+\Delta m^{2}} .
$$

This is a fair approximation to the result presented in [95] for the local theory. We have here a single Yukawa propagator while, in the local case, we have an infinite sum of such propagators.

Considering Eq. (C5) in Appendix C, we emphasize that this result is just an approximate one but holds when the nonlocal effects are seen to modify significantly the spectrum of the theory. The integral can be computed only when the entire function is fully specified, where we assume

$$
f\left(-p^{2}\right)=e^{-\frac{p^{2}}{M^{2}}} .
$$

Therefore, using dimensional regularization, the above integral can be rewritten in the form

$$
\begin{aligned}
u(0)= & -\frac{\left(N^{2}-1\right)^{2}}{(2 \pi)^{d} N} g^{2} \frac{2 \pi^{\frac{d}{2}}}{\Gamma(d / 2)} \frac{M^{d-2}}{\Delta m^{2}}\left[\frac{1}{2} \Gamma\left(\frac{d}{2}-1\right)\right. \\
& \left.-g\left(\frac{\Delta m^{2}}{M^{2}}\right)\right]
\end{aligned}
$$

where

$$
g(z)=\int_{0}^{\infty} d x x^{d-1} \frac{e^{-x^{2}}}{x^{2}+z e^{-\frac{x^{2}}{2}}} .
$$

For $z=\Delta m^{2} / M^{2} \ll 1$, we have the expansion

$$
\begin{aligned}
g(z)= & \frac{1}{2} \Gamma\left(\frac{d}{2}-1\right)-2^{\frac{d}{2}-3} 3^{2-\frac{d}{2}} \Gamma\left(\frac{d}{2}-2\right) z \\
& +2^{2-\frac{d}{2}} \Gamma\left(\frac{d}{2}-3\right) z^{2}+O\left(z^{3}\right) .
\end{aligned}
$$

It is interesting to point out that, using Eq. (45), the development parameter $z$ will be essentially given by the ratio $\mu^{2} / M^{2}$, with $\mu$ being the characteristic scale fixing the ground state of the theory and arising from as an integration constant. This just says to us that the infrared limit is the reliable one for our computation.

We can introduce the $\epsilon$ parameter as $d=4-\epsilon$ and we have

$$
\begin{aligned}
u(0)= & -\frac{\left(N^{2}-1\right)^{2}}{(2 \pi)^{4-\epsilon} N} g^{2} \frac{2 \pi^{2-\frac{\epsilon}{2}}}{\Gamma(2-\epsilon / 2)} M^{-\epsilon} z^{-1}\left[2^{-\frac{\epsilon}{2}-1} 3^{\frac{\epsilon}{3}} \Gamma\left(-\frac{\epsilon}{2}\right) z\right. \\
& \left.-2^{-\frac{\epsilon}{2}} \Gamma\left(-1-\frac{\epsilon}{2}\right) z^{2}+O\left(z^{3}\right)\right] .
\end{aligned}
$$

Finally, we expand in $\epsilon$ to get 


$$
\begin{aligned}
u(0)= & -\frac{\left(N^{2}-1\right)^{2}}{8 \pi^{2} N} g^{2} z^{-1}\left[\left(-\frac{1}{\epsilon}-\frac{\gamma}{2}\right) z-\left(\frac{2}{\epsilon}+\gamma-1\right) z^{2}\right. \\
& \left.+O\left(z^{3}\right)\right]+O(\epsilon) .
\end{aligned}
$$

We observe that the integral has a divergent part given by

$$
u_{\infty}(0)=\frac{\left(N^{2}-1\right)^{2}}{8 \pi^{2} N} g^{2} \frac{1}{\epsilon}\left(1+2 z+O\left(z^{2}\right)\right)
$$

that we reabsorb through a redefinition of the coupling, as the theory is renormalizable. Then, we write

$u(0)=\frac{\left(N^{2}-1\right)^{2}}{2 \pi N} \alpha_{s}\left[\frac{\gamma}{2}-(1-\gamma) z+O\left(z^{2}\right)\right]+O(\epsilon)$,

where $\alpha_{s}=g^{2} / 4 \pi$. Thus, the nonlocal Kugo-Ojima confinement condition yields the running coupling equation

$$
\frac{\left(N^{2}-1\right)^{2}}{2 \pi N^{2}} \alpha_{s}\left\{\frac{\gamma}{2}-(1-\gamma) \frac{\Delta m^{2}}{M^{2}}+O\left[\left(\frac{\Delta m^{2}}{M^{2}}\right)^{2}\right]\right\}=-1,
$$

where we have redefined $\alpha_{s} \rightarrow N \alpha_{s}$ to introduce the 't Hooft coupling. This is a confinement condition that depends on $\alpha_{s}$ in a highly nontrivial way. Working on shell, we have from Eq. (45)

$$
\Delta m^{2}=\mu^{2} \alpha_{s}^{\frac{1}{2}} \eta_{0} e^{-\eta_{1} \mu^{2} \alpha_{s}^{\frac{1}{2}} / M^{2}},
$$

with numerical constants

$$
\eta_{0}=(72 \pi)^{\frac{1}{2}} \frac{4 \pi^{2}}{K^{2}(i)} \frac{e^{-\pi}}{\left(1+e^{-\pi}\right)}
$$

and

$$
\eta_{1}=\frac{\pi^{2}}{4 K^{2}(i)}(2 \pi)^{\frac{1}{2}}
$$

The aim of this rewriting of the mass gap is that, in this way, we are able to explicitly show the dependence of the energy scale $\mu$ and the 't Hooft coupling $\alpha_{s}$ in the mass gap. This makes it clear that Eq. (59) is a highly nonlinear algebraic equation.

We observe that, due to the expansion with respect to $z=\Delta m^{2} / M^{2} \ll 1$, our conclusion can be trusted only in the infrared limit. This is enough for a proof of confinement. In this limit $\mu^{2} / M^{2} \rightarrow 0$, we can neglect the contribution coming from the exponential term in the mass gap and evaluate the corresponding beta function quite easily.

\section{NONLOCAL $\beta$ FUNCTION}

We derive the $\beta$ function using the following steps: (1) First, we differentiate Eq. (59) with respect to $l=\ln \left(\mu^{2} / M^{2}\right)$. (2) We extract the factor $e^{l}$ from Eq. (59) and substitute it into the differential equation obtained in step (1). (3) Finally, we extract $d \alpha_{s} / d l$ from step (1). Within the limit of our approximations, ${ }^{3}$ we obtain for the first step

$$
\begin{aligned}
& \beta_{0} \frac{d \alpha_{s}}{d l}\left(\frac{\gamma}{2}-(1-\gamma) e^{l} \sqrt{\alpha_{s}} \eta_{0}\right)+\beta_{0} \alpha_{s}(l)\left(-(1-\gamma) e^{l} \sqrt{\alpha_{s}(l)} \eta_{0}\right. \\
& \left.-\frac{1}{2 \sqrt{\alpha_{s}}}(1-\gamma) e^{l} \eta_{0} \frac{d \alpha_{s}}{d l}\right)=0
\end{aligned}
$$

with

$$
\beta_{0}=\frac{\left(N^{2}-1\right)^{2}}{2 \pi N^{2}} .
$$

From the second step, one has

$$
e^{l}=-\frac{\beta_{0} \alpha_{s}(l) \gamma+2}{2 \beta_{0} \alpha_{s}^{\frac{3}{2}}(l) \eta_{0}(\gamma-1)} .
$$

In the given approximations, from the third step, the $\beta$ function is found to be a very simple form,

$$
\frac{d \alpha_{s}}{d l}=\beta\left(\alpha_{s}\right)=-2 \frac{2+\beta_{0} \gamma \alpha_{s}}{6+\beta_{0} \gamma \alpha_{s}} \alpha_{s} .
$$

The coupling runs to infinity in the infrared, signaling confinement, and runs to zero in the ultraviolet signaling the asymptotic freedom.

The equation we obtained is amenable to an exact solution. In order to avoid formula clutter, we show here the leading order of an asymptotic expansion holding in the IR limit. One has

$$
\alpha_{s}(l) \sim \frac{\left(\beta_{0}^{2} \eta_{0}^{2}\right)^{-\frac{1}{3}}}{(1-\gamma)^{\frac{2}{3}}} \exp (-2 l / 3) .
$$

In the IR limit, $l \rightarrow-\infty$, and so $\alpha_{s}(l) \rightarrow \infty$. In the absence of a Landau ghost, this represents a confining theory.

It is important to notice that, in this way, we have consistently extended the renormalization group to the nonlocal field theories. It is necessary to emphasize that integration from 0 to infinity on the energy scale can take

\footnotetext{
${ }^{3}$ Note that the $\beta$ function we obtained is meaningful only in the IR limit where our approximations hold. The effect of the nonlocality appears to move the nontrivial fixed point to infinity, keeping confinement (no Landau pole). In the UV limit, we assume that asymptotic freedom holds, at least till the nonlocal mass scale that is assumed to be at very high energies.
} 


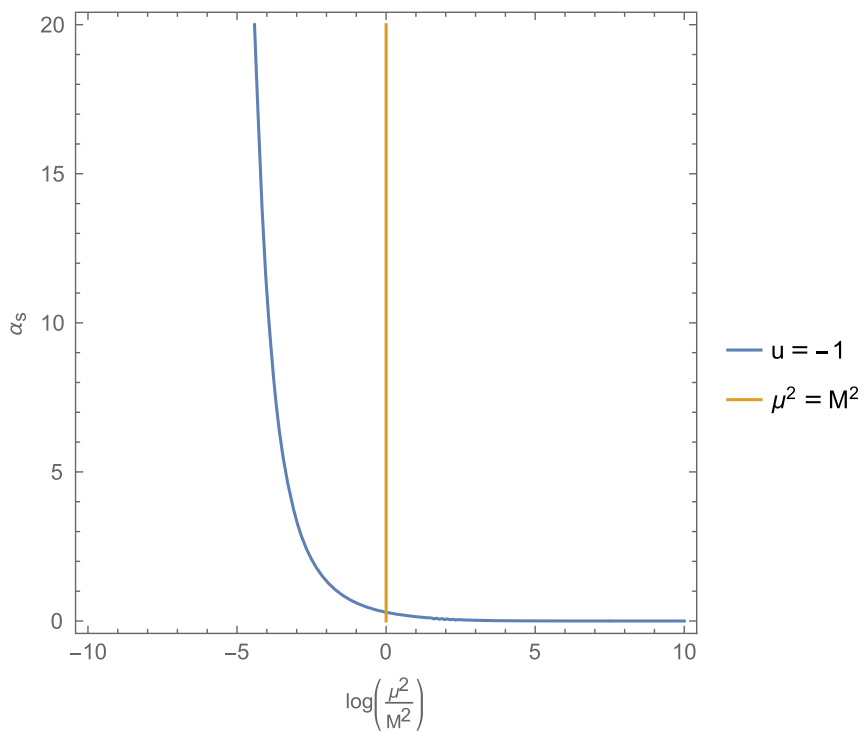

FIG. 1. Running coupling for nonlocal Yang-Mills theory with our approximations showing the behavior in the infrared. $\mu$ is the RGE scale and $M$ is the scale of nonlocality. The coupling is seen to run to infinity smoothly at lower energies. Although one can see asymptotic freedom beyond the nonlocal scale, our solution is not applicable for this energy range.

contributions also from the UV limit, modifying the beta function. On the other hand, in the UV limit we have omitted important contributions in the derivation of the $\beta$ function, so our results would need further improvement in this case. Anyway, we see that asymptotic freedom seems properly recovered but, due to our approximations, is mimicking the local case.

Finally, we can estimate the confinement scale by Eq. (67), which is

$$
\mu_{c}^{2} \sim\left(\frac{\alpha_{0}}{\alpha_{s}}\right)^{\frac{3}{2}} M^{2}
$$

where we have set

$$
\alpha_{0}=\left[(1-\gamma) \beta_{0} \eta_{0}\right]^{-\frac{2}{3}} .
$$

For $\mathrm{SU}(3)$, one has $\alpha_{0} \approx 0.2773$.

In Fig. 1, the running coupling is given with an indication of the starting of confinement limit at $\mu^{2}=M^{2}$. After that, the curve is seen to go to infinity steeply marking a confined regime of the theory, with the absence of Landau ghost.

\section{CONCLUSIONS AND DISCUSSIONS}

We investigated strongly coupled nonlocal gauge theory in four dimensions in the context of the confinement aspects of the theory. Concurrently we compared the results with that of the local theory and discussed the implications regarding realistic QCD-like scenarios in the infrared aspects. We presented the $\beta$ function of the theory and showed that the gauge condensation in the theory leads to dynamical generation of scales governed by the confinement scale and the scale of nonlocality. We summarize the main findings of our paper below:

(1) We derived the confinement conditions in the nonlocal gauge theories and showed that infinite-derivative nonlocal QFT in four dimensions provides confinement determined by the nonlocal scale $M$.

(2) We presented the renormalization group equations for the nonlocal gauge theory in the strongly coupled regimes and showed that the coupling runs to infinity in the low-energy limit, without encountering the problem of Landau ghosts. The theory restores conformal invariance in the UV with strong coupling due to dilution of the mass gap arising from the nonlocal effects.

(3) In the UV limit, our conclusion can be trusted only until a certain energy scale due to the approximation made. In any case, the effects in the integral that define the Kugo-Ojima $u$ function in Eq. (C5) are summed till the nonlocality scale. This yields finite contributions also in the IR limit and provides a proof of confinement, granted by the absence of the Landau ghost.

Our future studies will involve more detailed understanding of confinement and $\beta$-function analysis in the framework of infinite-derivative nonlocal gravity theories ${ }^{4}$ motivated from string field theory which provides a UVcomplete ghost-free, renormalizable approach to quantum gravity which is free from cosmological ${ }^{5}$ and black-hole singularities [30-47] but on the other hand leads to quadratic divergence-free, stable vacuum, no-Landau-pole and confomally invariant QFT valid up to infinite energy scales $[23,24,26-29,64] .^{6}$

\section{ACKNOWLEDGMENTS}

This work is supported in part by U.S. Department of Energy Grant No. DE-SC0012447 (N. O.).

\section{APPENDIX A: DYSON-SCHWINGER EQUATIONS AND BENDER-MILTON-SAVAGE TECHNIQUE}

In the following, we present the Bender-Milton-Savage technique [104]. This permits us to obtain the full hierarchy of Dyson-Schwinger equations in a partial differential equation (PDE) form.

\footnotetext{
${ }^{4}$ Whether such nonlocal factors may arise from any first principle was recently discussed in Ref. [98].

${ }^{5}$ For inflationary cosmology and predictions in the cosmic microwave background in nonlocal theories, see Refs. [93,99-102].

${ }^{6}$ See Ref. [103] for a recent conference on this topic.
} 
Let us consider the partition function for a scalar field

$$
Z[j]=\int[D \phi] e^{i S(\phi)+i \int d^{4} x j(x) \phi(x)} .
$$

For the 1P function we get

$$
\left\langle\frac{\delta S}{\delta \phi(x)}\right\rangle=j(x)
$$

where

$$
\langle\ldots\rangle=\frac{\int[D \phi] \ldots e^{i S(\phi)+i \int d^{4} x j(x) \phi(x)}}{\int[D \phi] e^{i S(\phi)+i \int d^{4} x j(x) \phi(x)}}
$$

After that, we can complete the procedure by setting $j=0$. Next step is to derive the above equation for the 1P function and dependent on $j$ to obtain the equation for the $2 \mathrm{P}$ function. We emphasize that the definition of the $\mathrm{nP}$ function is given by

$$
\left\langle\phi\left(x_{1}\right) \phi\left(x_{2}\right) \ldots \phi\left(x_{n}\right)\right\rangle=\frac{\delta^{n} \ln (Z[j])}{\delta j\left(x_{1}\right) \delta j\left(x_{2}\right) \ldots \delta j\left(x_{n}\right)} .
$$

Therefore,

$$
\frac{\delta G_{k}(\ldots)}{\delta j(x)}=G_{k+1}(\ldots, x)
$$

This means that, for a $\phi^{4}$ theory, one has

$$
S=\int d^{4} x\left[\frac{1}{2}(\partial \phi)^{2}-\frac{\lambda}{4} \phi^{4}\right],
$$

so that

$$
\partial^{2}\langle\phi\rangle+\lambda\left\langle\phi^{3}(x)\right\rangle=j(x)
$$

The following equation just holds:

$$
Z[j] \partial^{2} G_{1}^{(j)}(x)+\lambda\left\langle\phi^{3}(x)\right\rangle=j(x) .
$$

By the definition of the 1P function one gets

$$
Z[j] G_{1}^{(j)}(x)=\langle\phi(x)\rangle .
$$

Now we derive with respect to $j(x)$ to obtain

$$
Z[j]\left[G_{1}^{(j)}(x)\right]^{2}+Z[j] G_{2}^{(j)}(x, x)=\left\langle\phi^{2}(x)\right\rangle,
$$

and after another derivation step it is

$$
\begin{aligned}
& Z[j]\left[G_{1}^{(j)}(x)\right]^{3}+3 Z[j] G_{1}^{(j)}(x) G_{2}(x, x)+Z[j] G_{3}^{(j)}(x, x, x) \\
& \quad=\left\langle\phi^{3}(x)\right\rangle .
\end{aligned}
$$

Inserting it into Eq. (A7) yields

$$
\begin{aligned}
& \partial^{2} G_{1}^{(j)}(x)+\lambda\left[G_{1}^{(j)}(x)\right]^{3}+3 \lambda G_{2}^{(j)}(0) G_{1}^{(j)}(x)+G_{3}^{(j)}(0,0) \\
& \quad=Z^{-1}[j] j(x) .
\end{aligned}
$$

We realize that, by the effect of renormalization, a mass term appeared. We uncover here a term due to mass renormalization. Therefore, setting $j=0$, one puts the first Dyson-Schwinger equation into the differential form

$\partial^{2} G_{1}(x)+\lambda\left[G_{1}(x)\right]^{3}+3 \lambda G_{2}(0) G_{1}(x)+G_{3}(0,0)=0$.

By deriving Eq. (A12) again with respect to $j(y)$, we get

$$
\begin{aligned}
& \partial^{2} G_{2}^{(j)}(x, y)+3 \lambda\left[G_{1}^{(j)}(x)\right]^{2} G_{2}^{(j)}(x, y)+3 \lambda G_{3}^{(j)}(x, x, y) G_{1}^{(j)}(x) \\
& \quad+3 \lambda G_{2}^{(j)}(x, x) G_{2}^{(j)}(x, y)+G_{4}^{(j)}(x, x, x, y)=Z^{-1}[j] \delta^{4}(x-y) \\
& \quad+j(x) \frac{\delta}{\delta j(y)}\left(Z^{-1}[j]\right) .
\end{aligned}
$$

Inserting $j=0$, the equation for the $2 \mathrm{P}$ function takes the form

$$
\begin{gathered}
\partial^{2} G_{2}(x, y)+3 \lambda\left[G_{1}(x)\right]^{2} G_{2}(x, y)+3 \lambda G_{3}(0, y) G_{1}(x) \\
\quad+3 \lambda G_{2}(0) G_{2}(x, y)+G_{4}(0,0, y)=\delta^{4}(x-y) .
\end{gathered}
$$

This procedure can be iterated to any desired order providing all the hierarchy of Dyson-Schwinger equations in PDE form.

\section{APPENDIX B: DYSON-SCHWINGER EQUATIONS FOR 1P AND 2P FUNCTIONS}

In this Appendix, we derive the Dyson-Schwinger equations for the Yang-Mills field for the $1 \mathrm{P}$ and $2 \mathrm{P}$ functions.

For the $1 \mathrm{P}$ functions, after averaging the equations of motion, we get

$$
\begin{aligned}
& \square G_{1 \mu}^{(j) a}+g f^{a b c} e^{-\frac{1}{2} f(\square)}\left\langle\partial_{\nu}\left[e^{\frac{1}{2} f(\square)} \bar{A}_{\mu}^{b} e^{\frac{1}{2} f(\square)} A^{c \nu}\right]\right\rangle \\
& \quad+g f^{a b c} e^{-\frac{1}{2} f(\square)}\left\langle\left[e^{\frac{1}{2} f(\square)} A^{b \nu} e^{\frac{1}{2} f(\square)}\left(\partial_{\mu} A_{\nu}^{c}-\partial_{\nu} A_{\mu}^{c}\right)\right]\right\rangle \\
& g^{2} f^{a b c} f^{c d e} e^{-\frac{1}{2} f(\square)}\left\langle\left[e^{\frac{1}{2} f(\square)} A^{b \nu} e^{\frac{1}{2} f(\square)} A_{\nu}^{d} e^{\frac{1}{2} f(\square)} A_{\mu}^{e}\right]\right\rangle \\
& \quad+g f^{a b c} e^{\frac{1}{2} f(\square)}\left\langle\bar{c}^{b} \partial_{\mu} c^{c}\right\rangle=e^{\frac{1}{2} f(\square)} j_{\mu}^{a},
\end{aligned}
$$

and for the ghost

$$
-\square P_{1}^{(\eta) a}+g f^{a b c}\left\langle\left(e^{\frac{1}{2} f(\square)} A_{\mu}^{c}\right) \partial^{\mu} c^{b}\right\rangle=e^{\frac{1}{2} f(\square)} \eta^{a} .
$$


For our aims, we introduced the following 1P functions:

$$
\begin{aligned}
& G_{1 \mu}^{(j) a}(x)=Z^{-1}\left\langle A_{\mu}^{a}(x)\right\rangle, \\
& P_{1}^{(\eta) a}(x)==Z^{-1}\left\langle c^{a}(x)\right\rangle .
\end{aligned}
$$

The same should hold for $\bar{c}^{a}$ yielding $\bar{P}_{1}^{(\eta) a}(x)$. In order to evaluate the averages we consider the above definitions rewritten as

$$
\begin{aligned}
Z[j, \eta, \bar{\eta}] e^{\frac{1}{2} f(\square)} G_{1 \mu}^{(j) a}(x) & =\left\langle e^{\frac{1}{2} f(\square)} A_{\mu}^{a}(x)\right\rangle, \\
Z[j, \eta, \bar{\eta}] P_{1}^{(\eta) a}(x) & =\left\langle c^{a}(x)\right\rangle .
\end{aligned}
$$

The apexes $(j)$ and $(\eta)$ are there to remember the explicit dependence on the currents. Let us derive one time with respect to $j(x)$ on the first equation to get

$$
\begin{aligned}
& Z e^{\frac{1}{2} f(\square)} G_{2 \mu \nu}^{(j) a b}(x, x)+Z e^{\frac{1}{2} f(\square)} G_{1 \mu}^{(j) a}(x) e^{\frac{1}{2} f(\square)} G_{1 \nu}^{(j) b}(x) \\
& \quad=\left\langle e^{\frac{1}{2} f(\square)} A_{\mu}^{a}(x) e^{\frac{1}{2} f(\square)} A_{\nu}^{b}(x)\right\rangle .
\end{aligned}
$$

We apply the space-time derivative $\partial^{\nu}$ obtaining

$$
\begin{aligned}
& Z e^{\frac{1}{2} f(\square)} \partial^{\nu} G_{2 \mu \nu}^{(j) a b}(x, x)+Z e^{\frac{1}{2} f(\square)} \partial^{\nu} G_{1 \mu}^{(j) a}(x) e^{\frac{1}{2} f(\square)} G_{1 \nu}^{(j) b}(x) \\
& \quad=\left\langle e^{\frac{1}{2} f(\square)} \partial^{\nu} A_{\mu}^{a}(x) e^{\frac{1}{2} f(\square)} A_{\nu}^{b}(x)\right\rangle .
\end{aligned}
$$

We further derive Eq. (B5) with respect to $j^{c \nu}$ to get

$$
\begin{aligned}
& Z e^{\frac{1}{2} f(\square)} G_{2 \mu \nu}^{(j) a b}(x, x) e^{\frac{1}{2} f(\square)} G_{1}^{(j) \nu c}(x)+Z e^{\frac{1}{2} f(\square)} G_{3 \mu \nu}^{(j) a b c \nu}(x, x, x)+Z e^{\frac{1}{2} f(\square)} G_{1 \mu}^{(j) a}(x) e^{\frac{1}{2} f(\square)} G_{1 \nu}^{(j) b}(x) e^{\frac{1}{f} f(\square)} G_{1}^{(j) \nu c}(x) \\
& \quad+Z e^{\frac{1}{2} f(\square)} G_{2 \mu}^{(j) a c \nu}(x) e^{\frac{1}{2} f(\square)} G_{1 \nu}^{(j) b}+Z e^{\frac{1}{2} f(\square)} G_{2 \nu}^{(j) b c \nu}(x) e^{\frac{1}{2} f(\square)} G_{1 \mu}^{(j) a}(x)=\left\langle e^{\frac{1}{2} f(\square)} A_{\mu}^{a}(x) e^{\frac{1}{2} f(\square)} A_{\nu}^{b}(x) e^{\frac{1}{2} f(\square)} A^{c \nu}(x)\right\rangle,
\end{aligned}
$$

and we need to do the same for the ghost field. From Eq. (B3) we write

$$
Z[j, \eta, \bar{\eta}] P_{1}^{(\eta) a}(x)=\left\langle c^{a}(x)\right\rangle .
$$

After deriving with respect to $\partial_{\mu}$ and then with respect to $\bar{\eta}$, one has

$Z \bar{P}_{1}^{(\eta) b}(x) e^{\frac{1}{2} f(\square)} \partial^{\mu} P_{1}^{(\eta) a}(x)+Z \partial^{\mu} K_{2}^{(\eta) a b}(x, x)=\left\langle\bar{c}^{b} \partial^{\mu} c^{a}(x)\right\rangle$.

We have introduced a new $2 \mathrm{P}$ function defined as

$$
K_{2}^{(\eta) a b}(x, y)=\frac{1}{Z} \frac{\delta P_{1}^{(\eta) a}(x)}{\delta \eta^{b}(y)},
$$

and the other $2 \mathrm{P}$ function

$$
J_{2 \mu}^{(\eta, j) a b}(x, y)=\frac{1}{Z} \frac{\delta P_{1}^{(\eta) a}(x)}{\delta j^{b \mu}(y)} .
$$

So, by deriving Eq. (B8) with respect to $j^{b \mu}(x)$, the result is

$$
\begin{aligned}
& Z e^{\frac{1}{2} f(\square)} G_{1 \mu}^{(j) b}(x) \partial^{\mu} P_{1}^{(\eta) a}(x)+Z \partial^{\mu} J_{2 \mu}^{(\eta, j) a b}(x, x) \\
& \quad=\left\langle A_{\mu}^{b}(x) \partial^{\mu} c^{a}(x)\right\rangle .
\end{aligned}
$$

$$
\begin{aligned}
& \square G_{1 \mu}^{(j) a}+g f^{a b c} e^{-\frac{1}{2} f(\square)} \partial^{\nu}\left[e^{\frac{1}{2} f(\square)} G_{2 \mu \nu}^{(j) b c}(x, x)+e^{\frac{1}{2} f(\square)} G_{1 \mu}^{(j) b}(x) e^{\frac{1}{2} f(\square)} G_{1 \nu}^{(j) c}(x)\right]-g f^{a b c} e^{-\frac{1}{2} f(\square)}\left[e^{\frac{1}{2} f(\square)} \partial^{\nu} G_{2 \mu \nu}^{(j) b c}(x, x)\right. \\
& \left.\quad+e^{\frac{1}{2} f(\square)} \partial^{\nu} G_{1 \mu}^{(j) b}(x) e^{\frac{1}{2} f(\square)} G_{1 \nu}^{(j) c}(x)\right]-g f^{a b c} e^{-\frac{1}{2} f(\square)}\left[e^{\frac{1}{2} f(\square)} \partial_{\mu} G_{2 \nu}^{(j) b c \nu}(x, x)+e^{\frac{1}{2} f(\square)} \partial_{\mu} G_{1 \nu}^{(j) b}(x) e^{\frac{1}{2} f(\square)} G_{1}^{(j) c \nu}(x)\right] \\
& \quad+g^{2} f^{a b c} f^{c d e} e^{-\frac{1}{2} f(\square)}\left[e^{\frac{1}{2} f(\square)} G_{2 \mu \nu}^{(j) b d}(x, x) e^{\frac{1}{2} f(\square)} G_{1}^{(j) \nu e}(x)+e^{\frac{1}{2} f(\square)} \partial^{\nu} G_{3 \mu \nu}^{(j) b d e \nu}(x, x, x)\right. \\
& \left.\quad+e^{\frac{1}{2} f(\square)} G_{1 \mu}^{(j) b}(x) e^{\frac{1}{2} f(\square)} G_{1 \nu}^{(j) d}(x) e^{\frac{1}{2} f(\square)} G_{1}^{(j) \nu e}(x)+e^{\frac{1}{2} f(\square)} G_{2 \mu}^{(j) b e \nu}(x, x) e^{\frac{1}{2} f(\square)} G_{1 \nu}^{(j) d}(x)+e^{\frac{1}{2} f(\square)} G_{2 \nu}^{(j) d e \nu}(x, x) e^{\frac{1}{2} f(\square)} G_{1 \mu}^{(j) b}(x)\right] \\
& \quad-g f^{a b c} e^{\frac{1}{2} f(\square)}\left\{\bar{P}_{1}^{(\eta) b}(x) e^{\frac{1}{2} f(\square)}\left[\partial_{\mu} P_{1}^{(\eta) c}(x)\right]+\partial_{\mu}\left[K_{2}^{(\eta) b c}(x, x)\right]\right\}=e^{\frac{1}{2} f(\square)} j_{\mu}^{a} .
\end{aligned}
$$

The equation of the local theory given in [88] is easily obtained by setting the nonlocality factor to 1 , corresponding to the local limit $M \rightarrow \infty$. For the ghost field, it is

$$
-\square P_{1}^{(\eta) c}-g f^{a b c} e^{\frac{1}{2} f(\square)} G_{1 \mu}^{(j) a}(x) \partial^{\mu} P_{1}^{(\eta) b}(x)-g f^{a b c} \partial^{\mu} J_{2 \mu}^{(\eta, j) a b}(x, x)=e^{\frac{1}{2} f(\square)} \eta^{c} .
$$

After setting all the currents to zero, the Dyson-Schwinger equations for the 1P functions are obtained in the form given in the main text. 
From Eq. (B13), we derive it with respect to $j^{\lambda h}(y)$, obtaining

$$
\begin{aligned}
& \square G_{2 \mu \lambda}^{(j) a h}(x, y)+g f^{a b c} e^{-\frac{1}{2} f(\square)} \partial^{\nu}\left[e^{\frac{1}{2} f(\square)} G_{3 \mu \nu \lambda}^{(j) b c h}(x, x, y)+e^{\frac{1}{2} f(\square)} G_{2 \mu \lambda}^{(j) b h}(x, y) \times e^{\frac{1}{2} f(\square)} G_{1 \nu}^{(j) c}(x)++e^{\frac{1}{2} f(\square)} G_{1 \mu}^{(j) b}(x) e^{\frac{1}{2} f(\square)} G_{2 \nu \lambda}^{(j) c h}(x)\right] \\
& -g f^{a b c} e^{-\frac{1}{2} f(\square)}\left[e^{\frac{1}{2} f(\square)} \partial^{\nu} G_{2 \mu \nu \lambda}^{(j) b c h}(x, x, y)+e^{\frac{1}{2} f(\square)} \partial^{\nu} G_{2 \mu \lambda}^{(j) b h}(x, y) e^{\frac{1}{2} f(\square)} G_{1 \nu}^{(j) c}(x)+e^{\frac{1}{2} f(\square)} \partial^{\nu} G_{1 \mu}^{(j) b}(x) e^{\frac{1}{2} f(\square)} G_{2 \nu \lambda}^{(j) c h}(x, y)\right] \\
& -g f^{a b c} e^{-\frac{1}{2} f(\square)}\left[e^{\frac{1}{2} f(\square)} \partial_{\mu} G_{3 \nu \lambda}^{(j) b c h \nu}(x, x, y)+e^{\frac{1}{2} f(\square)} \partial_{\mu} G_{2 \nu \lambda}^{(j) b h}(x, y) e^{\frac{1}{2} f(\square)} G_{1}^{(j) c \nu}(x)+e^{\frac{1}{2} f(\square)} \partial_{\mu} G_{1 \nu}^{(j) b}(x) e^{\frac{1}{2} f(\square)} G_{2 \lambda}^{(j) c h \nu}(x, y)\right] \\
& +g^{2} f^{a b c} f^{c d e} e^{-\frac{1}{2} f(\square)}\left[e^{\frac{1}{2} f(\square)} G_{3 \mu \nu \lambda}^{(j) b d h}(x, x, y) e^{\frac{1}{2} f(\square)} G_{1}^{(j) \nu e}(x)+e^{\frac{1}{2} f(\square)} G_{2 \mu \nu}^{(j) b d}(x, x) e^{\frac{1}{2} f(\square)} G_{2 \lambda}^{(j) \nu e h}(x, y)\right. \\
& +e^{\frac{1}{2} f(\square)} \partial^{\nu} G_{4 \mu \nu \lambda}^{(j) b d e h \nu}(x, x, x, y)+e^{\frac{1}{2} f(\square)} G_{2 \mu \lambda}^{(j) b h}(x, y) e^{\frac{1}{2} f(\square)} G_{1 \nu}^{(j) d}(x) e^{\frac{1}{2} f(\square)} G_{1}^{(j) \nu e}(x) \\
& +e^{\frac{1}{2} f(\square)} G_{1 \mu}^{(j) b}(x) e^{\frac{1}{2} f(\square)} G_{2 \nu \lambda}^{(j) d h}(x, y) e^{\frac{1}{2} f(\square)} G_{1}^{(j) \nu e}(x)+e^{\frac{1}{2} f(\square)} G_{1 \mu}^{(j) b}(x) e^{e^{\frac{1}{2} f(\square)}} G_{1 \nu}^{(j) d}(x) e^{\frac{1}{2} f(\square)} G_{2 \lambda}^{(j) \nu e h}(x, y) \\
& +e^{\frac{1}{2} f(\square)} G_{3 \mu \lambda}^{(j) b e h \nu}(x, x, y) e^{\frac{1}{2} f(\square)} G_{1 \nu}^{(j) d}(x)+e^{\frac{1}{2} f(\square)} G_{2 \mu}^{(j) b e \nu}(x, x) e^{\frac{1}{2} f(\square)} G_{2 \nu \lambda}^{(j) d h}(x, y) \\
& \left.+e^{\frac{1}{2} f(\square)} G_{3 \nu \lambda}^{(j) d e h \nu}(x, x, y) e^{\frac{1}{2} f(\square)} G_{1 \mu}^{(j) b}(x)+e^{\frac{1}{2} f(\square)} G_{2 \nu}^{(j) d e \nu}(x, x) e^{\frac{1}{2} f(\square)} G_{2 \mu \lambda}^{(j) b h}(x, y)\right]-g f^{a b c} e^{\frac{1}{2} f(\square)}\left\{\bar{J}_{2 \lambda}^{(\eta, j) b h}(x, y) e^{\frac{1}{2} f(\square)}\left[\partial_{\mu} P_{1}^{(\eta) c}(x)\right]\right. \\
& \left.+\bar{P}_{1}^{(\eta) b}(x) e^{\frac{1}{2} f(\square)}\left[\partial_{\mu} J_{2 \lambda}^{(\eta) c h}(x, y)\right]+\partial_{\mu}\left[W_{3 \lambda}^{(\eta, j) b c h}(x, x, y)\right]\right\}=e^{\frac{1}{2} f(\square)} \delta^{a h} \eta_{\mu \lambda} \delta^{4}(x-y),
\end{aligned}
$$

after the introduction of the 3P function

$$
W_{3 \lambda}^{(\eta, j) a b c}(x, y, z)=Z^{-1} \frac{\delta K_{2}^{(\eta) a b}(x, y)}{\delta j^{\lambda c}(z)} .
$$

Similarly, starting from the 1P function for the ghost and deriving it with respect to $\eta^{h}(y)$, we get

$$
\begin{aligned}
& -\square K_{2}^{(\eta) c h}(x, y)-i g e^{\frac{1}{2} f^{a b c} f(\square)} L_{2 \mu}^{(\eta, j) a h}(x, y) \partial^{\mu} P_{1}^{(\eta) b}(x) \\
& -i g f^{a b c} e^{\frac{1}{2} f(\square)} G_{1 \mu}^{(j) a}(x) \partial^{\mu} K_{2}^{(\eta) b h}(x, y) \\
& -i g f^{a b c} \partial^{\mu} W_{3 \mu}^{(\eta, j) a b h}(x, x, y)=e^{\frac{1}{2} f(\square)} \delta^{c h} \delta^{4}(x-y) .
\end{aligned}
$$

We have introduced the $2 \mathrm{P}$ function

$$
L_{2 \mu}^{(\eta, j) a b}(x, y)=\frac{\delta G_{1}^{(j) a}(x)}{\delta \eta^{b}(y)}
$$

Deriving with respect to $j^{h \nu}(y)$, one has the equation for $J_{2}$ in the form

$$
\begin{aligned}
- & \square J_{2}^{(\eta) c h \nu}(x, y)-i g f^{a b c} e^{\frac{1}{2} f(\square)} G_{2 \mu \nu}^{(j) a h}(x, y) \partial^{\mu} P_{1}^{(\eta) b}(x) \\
& -i g f^{a b c} e^{\frac{1}{2} f(\square)} G_{1 \mu}^{(j) a}(x) \partial^{\mu} J_{2}^{(\eta, j) b h \nu}(x, y) \\
& -i g f^{a b c} \partial^{\mu} J_{3 \mu}^{(\eta, j) a b h}(x, x, y)=0
\end{aligned}
$$

with the introduction of the 3P function

$$
J_{3 \mu}^{(\eta, j) a b c}(x, y, z)=\frac{\delta J_{2 \mu}^{(\eta, j) a b}(x, y)}{\delta j^{c \mu}(z)}
$$

We can recover the equations in the main text after setting all the currents to zero.

\section{APPENDIX C: CONFINEMENT IN LOCAL YANG-MILLS THEORY}

The approach given in the main text is straightforwardly obtained by the analysis performed in Ref. [95]. The $u$ function can be obtained by observing that

$$
\int d^{4} x e^{i p x}\left\langle D_{\mu} \bar{c}^{a}(x), D_{\nu} c^{b}(0)\right\rangle=-\delta^{a b} \frac{p_{\mu} p_{\nu}}{k^{2}}+\frac{\left(N^{2}-1\right)^{2}}{2 N} g^{2} \delta^{a b}\left(\delta_{\mu \nu}-\frac{p_{\mu} p_{\nu}}{p^{2}}\right) \int \frac{d^{4} p^{\prime}}{(2 \pi)^{4}} K_{2}\left(p-p^{\prime}\right) G_{2}\left(p^{\prime}\right) .
$$

For local Yang-Mills theory, the propagators take the form

$$
K_{2}(p)=-\frac{1}{p^{2}+i \epsilon}
$$

for the ghost field and

$$
G_{2}(p)=\frac{\pi^{3}}{4 K^{3}(i)} \sum_{n=0}^{\infty} \frac{e^{-\left(n+\frac{1}{2}\right) \pi}}{1+e^{-(2 n+1) \pi}}(2 n+1)^{2} \frac{1}{p^{2}-m_{n}^{2}+i \epsilon}
$$

for the gauge field, with the mass spectrum 


$$
m_{n}=(2 n+1) \frac{\pi}{2 K(i)}\left(\frac{N g^{2}}{2}\right)^{\frac{1}{4}} \mu,
$$

where $K(i)$ is the complete elliptical integral of the first kind and $\mu$ is one of the integration constants of the theory. This is an approximate solution as we have neglected any mass shift arising from renormalization. This will yield for the confinement condition by Kugo-Ojima

$$
\begin{aligned}
u(0)= & -\frac{\left(N^{2}-1\right)^{2}}{2 N} g^{2} \int \frac{d^{4} p}{(2 \pi)^{4}} \frac{1}{p^{2}+i \epsilon} \frac{\pi^{3}}{4 K^{3}(-1)} \\
& \times \sum_{n=0}^{\infty} \frac{e^{-\left(n+\frac{1}{2}\right) \pi}}{1+e^{-(2 n+1) \pi}}(2 n+1)^{2} \frac{1}{p^{2}-m_{n}^{2}+i \epsilon}=-1 .
\end{aligned}
$$

[1] J. W. Moffat, Phys. Rev. D 41, 1177 (1990).

[2] D. Evens, J. W. Moffat, G. Kleppe, and R. P. Woodard, Phys. Rev. D 43, 499 (1991).

[3] E. T. Tomboulis, arXiv:hep-th/9702146.

[4] J. W. Moffat, arXiv:1104.5706.

[5] E. T. Tomboulis, Phys. Rev. D 92, 125037 (2015).

[6] G. Kleppe and R.P. Woodard, Nucl. Phys. B388, 81 (1992).

[7] E. Witten, Nucl. Phys. B268, 253 (1986).

[8] V. A. Kostelecky and S. Samuel, Nucl. Phys. B336, 263 (1990).

[9] V. A. Kostelecky and S. Samuel, Phys. Lett. B 207, 169 (1988).

[10] P. G. Freund and M. Olson, Phys. Lett. B 199, 186 (1987).

[11] P. G. Freund and E. Witten, Phys. Lett. B 199, 191 (1987).

[12] L. Brekke, P. G. Freund, M. Olson, and E. Witten, Nucl. Phys. B302, 365 (1988).

[13] P. H. Frampton and Y. Okada, Phys. Rev. D 37, 3077 (1988).

[14] T. Biswas, M. Grisaru, and W. Siegel, Nucl. Phys. B708, 317 (2005).

[15] A. A. Tseytlin, Phys. Lett. B 363, 223 (1995).

[16] W. Siegel, arXiv:hep-th/0309093.

[17] G. Calcagni and L. Modesto, J. Phys. A 47, 355402 (2014).

[18] L. Modesto, Phys. Rev. D 86, 044005 (2012).

[19] L. Modesto, arXiv:1202.0008.

[20] L. Modesto, M. Piva, and L. Rachwal, Phys. Rev. D 94, 025021 (2016).

[21] L. Modesto, L. Rachwał, and I. L. Shapiro, Eur. Phys. J. C 78, 555 (2018).

[22] N. V. Krasnikov, Theor. Math. Phys. 73, 1184 (1987).

[23] T. Biswas and N. Okada, Nucl. Phys. B898, 113 (2015).

[24] A. Ghoshal, A. Mazumdar, N. Okada, and D. Villalba, Phys. Rev. D 97, 076011 (2018).
This integral can be performed using a continuation in the complex plane, moving to Euclidean. It is divergent and can be evaluated e.g., by dimensional regularization. This will yield the beta function in closed form for the theory, holding both in the UV and IR, as

$$
\beta_{\mathrm{YM}}=-\beta_{0} \frac{\alpha_{s}^{2}}{1-\frac{1}{2} \beta_{0} \alpha_{s}},
$$

with $\beta_{0}=\left(N^{2}-1\right)^{2} / 8 \pi N$. This beta function grants confinement in the IR. In the UV we recover the asymptotic freedom as expected.
[25] L. Buoninfante, G. Lambiase, and A. Mazumdar, Nucl. Phys. B944, 114646 (2019).

[26] A. Ghoshal, Int. J. Mod. Phys. A 34, 1950130 (2019).

[27] L. Buoninfante, A. Ghoshal, G. Lambiase, and A. Mazumdar, Phys. Rev. D 99, 044032 (2019).

[28] M. Frasca and A. Ghoshal, Classical Quant. Grav. 38, 175013 (2021).

[29] M. Frasca and A. Ghoshal, J. High Energy Phys. 07 (2021) 226.

[30] T. Biswas, E. Gerwick, T. Koivisto, and A. Mazumdar, Phys. Rev. Lett. 108, 031101 (2012).

[31] T. Biswas, A. Conroy, A. S. Koshelev, and A. Mazumdar, Classical Quant. Grav. 31, 015022 (2014); 31, 159501(E) (2014).

[32] V. P. Frolov, A. Zelnikov, and T. de Paula Netto, J. High Energy Phys. 06 (2015) 107.

[33] V. P. Frolov and A. Zelnikov, Phys. Rev. D 93, 064048 (2016).

[34] A. S. Koshelev, J. Marto, and A. Mazumdar, Phys. Rev. D 98, 064023 (2018).

[35] A. S. Koshelev and A. Mazumdar, Phys. Rev. D 96, 084069 (2017).

[36] L. Buoninfante, A. S. Koshelev, G. Lambiase, and A. Mazumdar, J. Cosmol. Astropart. Phys. 09 (2018) 034.

[37] A. S. Cornell, G. Harmsen, G. Lambiase, and A. Mazumdar, Phys. Rev. D 97, 104006 (2018).

[38] L. Buoninfante, A. S. Koshelev, G. Lambiase, J. Marto, and A. Mazumdar, J. Cosmol. Astropart. Phys. 06 (2018) 014.

[39] L. Buoninfante, G. Harmsen, S. Maheshwari, and A. Mazumdar, Phys. Rev. D 98, 084009 (2018).

[40] S. Abel, L. Buoninfante, and A. Mazumdar, J. High Energy Phys. 01 (2020) 003.

[41] L. Buoninfante, G. Lambiase, Y. Miyashita, W. Takebe, and M. Yamaguchi, Phys. Rev. D 101, 084019 (2020). 
[42] T. Biswas, A. Mazumdar, and W. Siegel, J. Cosmol. Astropart. Phys. 03 (2006) 009.

[43] T. Biswas, R. Brandenberger, A. Mazumdar, and W. Siegel, J. Cosmol. Astropart. Phys. 12 (2007) 011.

[44] T. Biswas, T. Koivisto, and A. Mazumdar, J. Cosmol. Astropart. Phys. 11 (2010) 008.

[45] T. Biswas, A. S. Koshelev, A. Mazumdar, and S. Y. Vernov, J. Cosmol. Astropart. Phys. 08 (2012) 024.

[46] A. S. Koshelev and S. Y. Vernov, Phys. Part. Nucl. 43, 666 (2012).

[47] A. S. Koshelev, J. Marto, and A. Mazumdar, J. Cosmol. Astropart. Phys. 02 (2019) 020.

[48] K. G. Wilson, Phys. Rev. B 4, 3174 (1971).

[49] K. G. Wilson, Phys. Rev. B 4, 3184 (1971).

[50] D. J. Gross and F. Wilczek, Phys. Rev. Lett. 30, 1343 (1973).

[51] H. D. Politzer, Phys. Rev. Lett. 30, 1346 (1973).

[52] D. J. E. Callaway, Phys. Rep. 167, 241 (1988).

[53] S. Weinberg, in General Relativity: An Einstein Centenary Survey, edited by S. W. Hawking and W. Israel (Cambridge University Press, Cambridge, England, 1979), pp. 790-831.

[54] D. F. Litim, Phil. Trans. R. Soc. A 369, 2759 (2011).

[55] D. F. Litim, AIP Conf. Proc. 841, 322 (2006).

[56] M. Niedermaier, Classical Quant. Grav. 24, R171 (2007).

[57] M. Niedermaier and M. Reuter, Living Rev. Relativity 9, 5 (2006).

[58] R. Percacci, arXiv:0709.3851.

[59] D. F. Litim, Proc. Sci., QG-Ph (2007) 024.

[60] M. Reuter and F. Saueressig, New J. Phys. 14, 055022 (2012).

[61] D. F. Litim and F. Sannino, J. High Energy Phys. 12 (2014) 178.

[62] G. F. Giudice, G. Isidori, A. Salvio, and A. Strumia, J. High Energy Phys. 02 (2015) 137.

[63] G. M. Pelaggi, A. D. Plascencia, A. Salvio, F. Sannino, J. Smirnov, and A. Strumia, Phys. Rev. D 97, 095013 (2018).

[64] A. Ghoshal, A. Mazumdar, N. Okada, and D. Villalba, Phys. Rev. D 104, 015003 (2021).

[65] J. B. Kogut and M. A. Stephanov, The Phases of Quantum Chromodynamics: From Confinement to Extreme Environments (Cambridge University Press, Cambridge, England, 2004).

[66] T. Kugo and I. Ojima, Phys. Lett. 73B, 459 (1978).

[67] T. Kugo and I. Ojima, Prog. Theor. Phys. Suppl. 66, 1 (1979).

[68] K. Nishijima, Int. J. Mod. Phys. A 09, 3799 (1994).

[69] K. Nishijima, Int. J. Mod. Phys. A 10, 3155 (1995).

[70] M. Chaichian and K. Nishijima, Eur. Phys. J. C 22, 463 (2001).

[71] M. Chaichian and K. Nishijima, Eur. Phys. J. C 47, 737 (2006).

[72] K. Nishijima and A. Tureanu, Eur. Phys. J. C 53, 649 (2008).

[73] N. Seiberg and E. Witten, Nucl. Phys. B431, 484 (1994).

[74] N. Seiberg and E. Witten, Nucl. Phys. B426, 19 (1994); B430, 485(E) (1994).
[75] M. Chaichian and T. Kobayashi, Phys. Lett. B 481, 26 (2000).

[76] V. N. Gribov, Nucl. Phys. B139, 1 (1978).

[77] D. Zwanziger, Nucl. Phys. B323, 513 (1989).

[78] I. L. Bogolubsky, E. M. Ilgenfritz, M. Muller-Preussker, and A. Sternbeck, Proc. Sci., LAT2007 (2007) 290 [arXiv:0710.1968].

[79] A. Cucchieri and T. Mendes, Proc. Sci., LAT2007 (2007) 297 [arXiv:0710.0412].

[80] O. Oliveira, P. J. Silva, E. M. Ilgenfritz, and A. Sternbeck, Proc. Sci., LAT2007 (2007) 323 [arXiv:0710.1424].

[81] B. Lucini, M. Teper, and U. Wenger, J. High Energy Phys. 06 (2004) 012.

[82] Y. Chen et al., Phys. Rev. D 73, 014516 (2006).

[83] J. M. Cornwall, Phys. Rev. D 26, 1453 (1982).

[84] J. M. Cornwall, J. Papavassiliou, and D. Binosi, The Pinch Technique and Its Applications to Non-Abelian Gauge Theories (Cambridge University Press, Cambridge, England, 2010).

[85] D. Dudal, J. A. Gracey, S. P. Sorella, N. Vandersickel, and H. Verschelde, Phys. Rev. D 78, 065047 (2008).

[86] M. Frasca, Phys. Lett. B 670, 73 (2008).

[87] M. Frasca, Mod. Phys. Lett. A 24, 2425 (2009).

[88] M. Frasca, Eur. Phys. J. Plus 132, 38 (2017); 132, 242(E) (2017).

[89] A. Deur, S. J. Brodsky, and G. F. de Teramond, Prog. Part. Nucl. Phys. 90, 1 (2016).

[90] A. Deur, Eur. Phys. J. C 77, 412 (2017).

[91] R. Pius and A. Sen, J. High Energy Phys. 10 (2016) 024; 09 (2018) 122(E).

[92] F. Briscese and L. Modesto, Phys. Rev. D 99, 104043 (2019).

[93] A. S. Koshelev and A. Tokareva, Phys. Rev. D 102, 123518 (2020).

[94] A. S. Koshelev and A. Tokareva, Phys. Rev. D 104, 025016 (2021).

[95] M. Chaichian and M. Frasca, Phys. Lett. B 781, 33 (2018).

[96] M. E. Peskin and D. V. Schroeder, An Introduction to Quantum Field Theory (Perseus Books, Reading, MA, 1995).

[97] S. P. Klevansky, Rev. Mod. Phys. 64, 649 (1992).

[98] L. Buoninfante, arXiv:2105.08167.

[99] A. S. Koshelev, K. Sravan Kumar, and P. Vargas Moniz, Phys. Rev. D 96, 103503 (2017).

[100] K. Sravan Kumar and L. Modesto, arXiv:1810.02345.

[101] A. S. Koshelev, K. S. Kumar, and A. A. Starobinsky, Int. J. Mod. Phys. D 29, 2043018 (2020).

[102] A. S. Koshelev, K. Sravan Kumar, A. Mazumdar, and A. A. Starobinsky, J. High Energy Phys. 06 (2020) 152.

[103] in Proceedings of the International Online Conference on Quantum Gravity, Higher Derivatives and Nonlocalities, 2021, https://www.qgrav2021.com/.

[104] C. M. Bender, K. A. Milton, and V. M. Savage, Phys. Rev. D 62, 085001 (2000). 